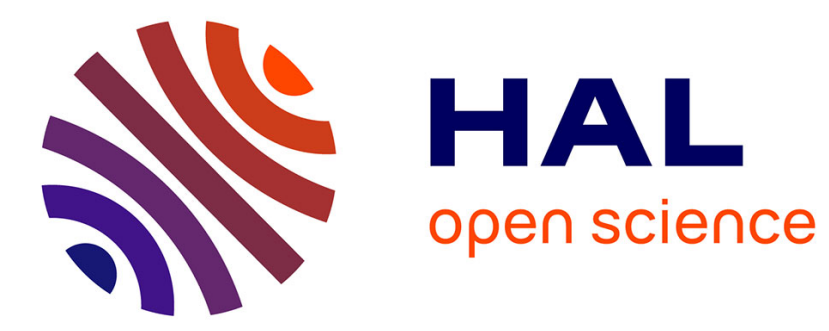

\title{
Recherches sur la résistance des sols aux maladies. XII. - Activité respiratoire dans un sol résistant et un sol sensible aux fusarioses vasculaires enrichis en glucose
}

Claude Alabouvette, Yvonne Couteaudier, Jean Louvet

\section{- To cite this version:}

Claude Alabouvette, Yvonne Couteaudier, Jean Louvet. Recherches sur la résistance des sols aux maladies. XII. - Activité respiratoire dans un sol résistant et un sol sensible aux fusarioses vasculaires enrichis en glucose. Agronomie, 1985, 5 (1), pp.69-72. hal-00884735

\section{HAL Id: hal-00884735 \\ https://hal.science/hal-00884735}

Submitted on 1 Jan 1985

HAL is a multi-disciplinary open access archive for the deposit and dissemination of scientific research documents, whether they are published or not. The documents may come from teaching and research institutions in France or abroad, or from public or private research centers.
L'archive ouverte pluridisciplinaire HAL, est destinée au dépôt et à la diffusion de documents scientifiques de niveau recherche, publiés ou non, émanant des établissements d'enseignement et de recherche français ou étrangers, des laboratoires publics ou privés. 


\title{
Recherches sur la résistance des sols aux mala- dies. XII. - Activité respiratoire dans un sol résistant et un sol sensible aux fusarioses vascu- laires enrichis en glucose
}

\author{
Claude ALABOUVETTE, Yvonne COUTEAUDIER \& Jean LOUVET \\ I.N.R.A., Station de Recherches sur la Flore pathogène dans le Sol, 17, rue Sully, F 21034 Dijon Cedex
}

Des travaux antérieurs ont permis de montrer que la germination des chlamydospores et le développement saprophytique des Fusarium spp. nécessitent un apport d'énergie plus important dans le sol résistant de Châteaurenard que dans le sol sensible d'Ouroux. Ces résultats suggérant que la compétition pour les éléments nutritifs est plus intense en terre résistante qu'en terre sensible, nous avons étudié l'activité respiratoire de ces 2 sols en réponse à un apport de glucose, afin d'apprécier leurs niveaux de biomasse et d'activité microbienne. Les résultats acquis montrent que, pour toutes les concentrations de glucose étudiées, le taux respiratoire initial est 2 à 4 fois plus élevé dans le sol résistant (tabl. 1), ce qui indique que la biomasse microbienne du sol résistant est plus importante que celle du sol sensible. L'étude cinétique du dégagement de $\mathrm{CO}_{2}$ après apport de glucose à la concentration de $1 \mathrm{mg} / \mathrm{g}$ (fig. 2) montre que les microorganismes se multiplient plus rapidement et plus intensément dans le sol résistant au cours des 12 premières heures qui suivent l'apport d'élément nutritif. Mais au-delà, le taux respiratoire diminue brusquement et se stabilise à un niveau extrêmement faible indiquant l'arrêt de la croissance microbienne. Au contraire, en terre sensible, le taux respiratoire augmente plus lentement, mais demeure à un niveau élevé pendant au moins $60 \mathrm{~h}$, ce qui prouve que l'état nutritionnel du sol continue de permettre le développement des microorganismes. Ces résultats démontrent que, dans le sol résistant, les chlamydospores de Fusarium disposent d'un laps de temps très limité pour germer et se développer en direction de la racine. Ainsi, un niveau de biomasse élevé détermine dans le sol résistant une compétition nutritive particulièrement intense qui limite considérablement les possibilités de développement des microorganismes et en particulier celui des Fusarium pathogènes.

Mots clés additionnels : Biomasse, activité microbienne, dioxyde de carbone, compétition nutritive. to a wilt-suppressive and a wilt-conductive soil.

Previous results indicated that to stimulate chlamydospore germination and saprophytic growth of Fusarium spp., it was necessary to add a greater concentration of glucose into the Châteaurenard suppressive soil than into the Ouroux conducive soil. These results suggested that competition for nutrients was greater in suppressive than in conducive soil. We therefore studied the evolution of $\mathrm{CO}_{2}$ after addition of glucose to the respective soils, in order to estimate the relative amounts of biomass and microbial activity of these two soils. The initial respiration rate was 2 to 4 times higher in the suppressive than in the conducive soil (tabl. 1), indicating that the initial size of the biomass was greater in the suppressive soil. The kinetics of $\mathrm{CO}_{2}$ release after adding glucose at $1 \mathrm{mg} / \mathrm{g}$ soil (fig. 2) showed further that the microbial activity started sooner and increased faster in the suppressive than in the conducive soil. However by $12 \mathrm{~h}$ after addition of glucose, the respiration rate decreased quickly and stabilized at a low level, indicating that some limiting factors prevented microbial growth. In the conducive soil, on the other hand, the respiration rate increased more slowly but remained high for a long period, indicating that the nutritional status of the soil could support microbial activity for a least $60 \mathrm{~h}$ after addition of glucose. These results suggest that in the suppressive soil the chlamydospores of Fusarium spp. had a shorter time after addition of glucose to germinate and reach the root surface. Thus, the larger and more responsive biomass acts as a greater nutrient sink for carbon and energy needed by the chlamydospores. This more intense competition for nutrients limits the chance of development for any given microorganisms, especially for pathogenic Fusarium.

Additional key words : Biomass, microbial activity, carbon dioxide, competition for nutrients. 


\section{INTRODUCTION}

Au cours de travaux précédents, nous avons démontré que la germination des chlamydospores et le développement saprophytique des Fusarium spp. nécessitent un apport d'énergie plus important dans le sol résistant de Châteaurenard que dans le sol d'Ouroux (AlabouveTte et al., 1985). Ces résultats ont été interprétés par rapport aux phénomènes généraux de fongistase et de compétition nutritive qui, dans tous les sols, contrôlent la mise en activité des organes de conservation et le développement des microorganismes (LOCKWOOD, 1981). Ainsi, la compétition pour les sources d'énergie, plus intense dans le sol résistant que dans le sol sensible, conditionnerait le niveau de réceptivité de ces sols aux fusarioses vasculaires. Deux paramètres essentiels déterminent l'intensité de la compétition pour un élément donné : d'une part, la quantité de nutriment disponible et, d'autre part, la masse de microorganismes capables de l'utiliser immédiatement. Nous avons montré dans l'article précédent qu'un apport de glucose réalisé à la même concentration n'a pas les mêmes effets sur la population de Fusarium spp. dans les 2 sols étudiés ; il est nécessaire d'apporter 10 fois plus d'énergie en terre résistante pour y observer le même taux de multiplication des populations fusariennes qu'en terre sensible (AlABOUVETTE et al., 1985). Ces résultats suggèrent donc que la biomasse microbienne capable d'utiliser cet élément nutritif est plus importante ou plus active dans la terre résistante et que par conséquent, la quantité d'énergie mise à la disposition de chaque propagule de Fusarium spp. y est plus limitée qu'en terre sensible. C'est afin de vérifier cette hypothèse que nous avons fait appel à une technique permettant d'apprécier l'activité microbienne des sols.

Les méthodes microbiologiques de dénombrement utilisées auparavant (ALABOUVETTE, 1983) ne renseignent pas sur l'activité des microorganismes dénombrés. C'est pourquoi, au cours des dernières années, diverses méthodes ont été proposées afin d'effectuer une estimation plus ou moins directe de la biomasse des sols. Deux d'entre elles (JENKINSON \& POWELSON, 1976 ; ANDERSON \& DOMSCH, 1978) sont basées sur la mesure de l'activité respiratoire des sols. Nous avons choisi d'adapter à notre étude la techrique décrite par ANDERSON \& DOMSCH (1978) car elle permet de suivre l'évolution au cours du temps de la réponse respiratoire des sols enrichis en glucose. Notre but n'est pas seulement de comparer le niveau de biomasse des sols résistant et sensible mais d'étudier l'activité de leurs microflores en réponse à un apport nutritif déterminé.

\section{MATÉRIEL ET MÉTHODES}

Les caractéristiques du sol résistant de Châteaurenard et du sol sensible d'Ouroux ont été décrites précédemment (ALABOUVETTE et al., 1980b). La méthode d'estimation de la biomasse microbienne décrite par ANDERSON \& DOMSCH (1978) consiste à déterminer le taux respiratoire initial maximum enregistré à la suite d'un apport de glucose en quantité non limitante. Nous avons modifié cette technique en employant du glucose marqué au ${ }^{14} \mathrm{C}$ et en utilisant des concentrations de glucose relativement faibles $(0,1,0,5$ et $1 \mathrm{mg} / \mathrm{g}$ de sol sec) (AlABOUVETTE, 1983).

Les sols préalablement tamisés à $6 \mathrm{~mm}$ sont humidifiés et incubés à la température du laboratoire pendant 3 semaines. Des échantillons d'un poids sec de $50 \mathrm{~g}$ sont alors déposés dans des flacons de $250 \mathrm{ml}$. Leur humidité est ajustée de telle sorte qu'elle corresponde à un potentiel hydrique de $-0,1$ bar après l'apport nutritif. Le glucose est apporté en solution à raison de $3 \mathrm{ml}$ par échantillon. La proportion de molécules marquées au ${ }^{14} \mathrm{C}$ est établie de manière à obtenir une précision satisfaisante au moment de la lecture au scintillateur ; elle correspond à $3 \mu$ curies $/ g$ de sol. Une pompe à galets assure un flux continu d'air ambiant privé de $\mathrm{CO}_{2}$ qui balaie les échantillons de sol. Le $\mathrm{CO}_{2}$ dégagé est recueilli dans un pilulier contenant $10 \mathrm{ml}$ de soude $0,2 \mathrm{~N}$ et le dosage est effectué au scintillateur.

\section{RÉSULTATS}

Pour chacun des apports de glucose, le taux respiratoire initial estimé entre 0 et $5 \mathrm{~h}$ (tabl. 1 , fig. 1 et 2 ) est 2 à 4 fois plus élevé en terre résistante qu'en terre sensible. D'autre part, la cinétique du dégagement de $\mathrm{CO}_{2}$ (fig. 1 et 2 ) est fondamentalement différente entre les 2 terres.

\section{DISCUSSION}

Dans le sol résistant la biomasse microbienne est plus importante que dans le sol sensible puisque le taux respiratoire initial après apport de glucose y est toujours plus élevé. Ceci est en concordance avec le résultat des analyses microbiologiques effectuées antérieurement (ALABOUVETTE, 1983).

L'évolution ultérieure du taux respiratoire, donc de l'activité microbienne, est également très différente dans les 2 sols. Pour les concentrations de glucose de 0,5 et $1 \mathrm{mg} / \mathrm{g}$ (fig. 1 et 2 ), le dégagement de $\mathrm{CO}_{2}$ aug mente plus vite et atteint sa valeur maximum plus tôt en terre résistante (6-12 h) qu'en terre sensible (12$23 \mathrm{~h}$ ). Il décroît également plus vite. Le développement microbien induit par un même apport énergétique est donc de plus courte durée dans le sol résistant. Le retour de la microflore à l'état de repos, qui caractérise les sols à l'équilibre et est une conséquence des phénomènes de compétition (MANGENOT \& DIEM, 1979 ; LOCKWOOD, 1981), s’y produit beaucoup plus tôt.

\section{TABLEAU}

Taux respiratoire initial de la terre résistante et de la terre sensible après apports de glucose (nanomoles $\mathrm{CO}_{2} / \mathrm{g} / \mathrm{h}$ ). Initial respiratory rate of suppressive and conducive soil after addition of glucose (nmole $\mathrm{CO}_{2} / \mathrm{g} / \mathrm{h}$ ).

\begin{tabular}{lccc}
\hline \hline & \multicolumn{3}{c}{ Glucose (mg/g de sol) } \\
\cline { 2 - 4 } & 0,1 & 0,5 & 1 \\
\hline Terre résistante & 29 & 94 & 120 \\
Terre sensible & 14 & 24 & 40 \\
\hline
\end{tabular}



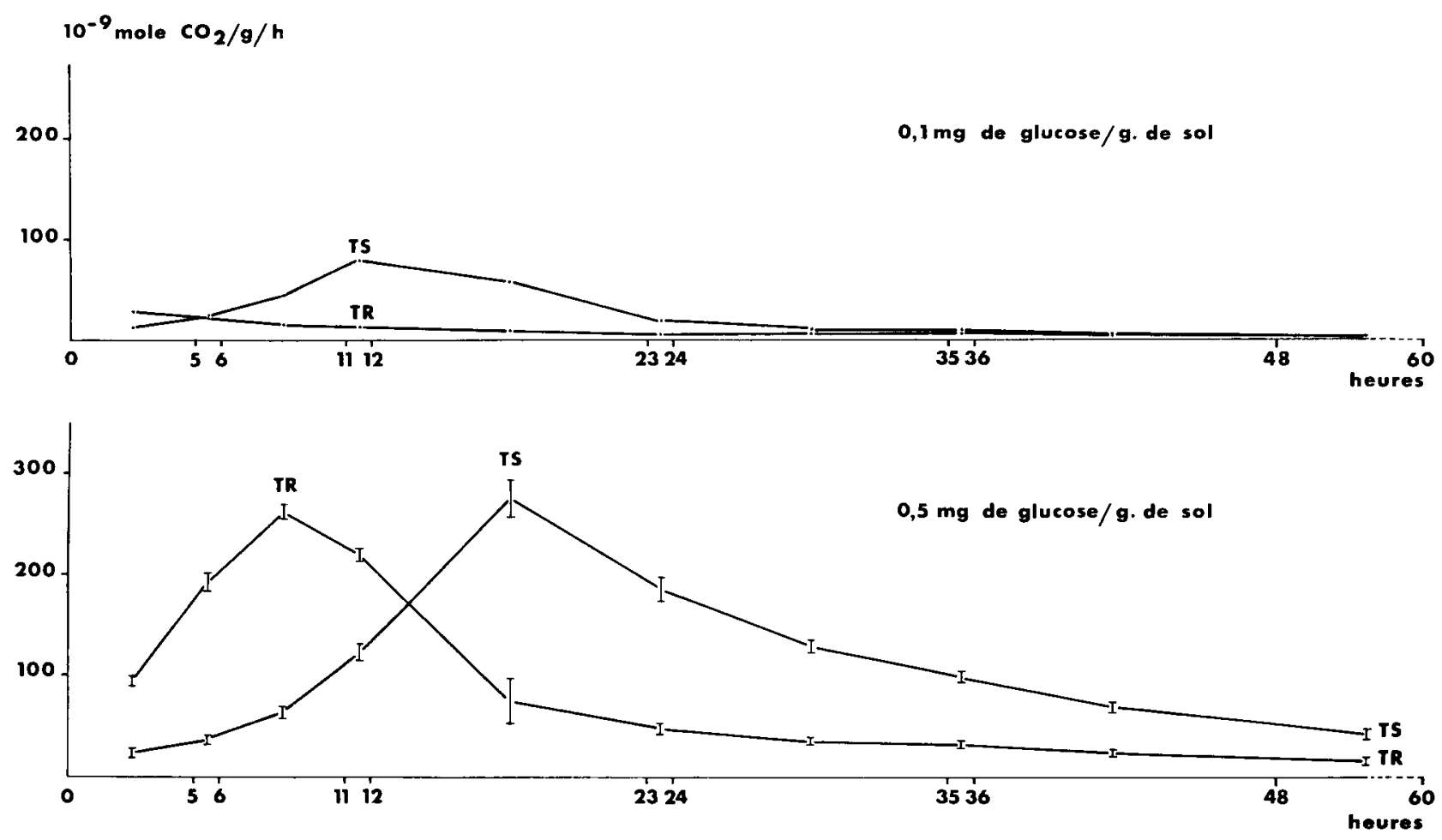

\section{Figure 1}

Cinétique du dégagement de $\mathrm{CO}_{2}$ par une terre résistante (TR) et par une terre sensible (TS) après addition de glucose $(0,1$ et $0,5 \mathrm{mg} / \mathrm{g}$ de terre).
Kinetics of $\mathrm{CO}_{2}$ release from suppressive (TR) and conducive (TS) soil after addition of 0.1 and $0.5 \mathrm{mg}$ of glucose per $\mathrm{g}$.

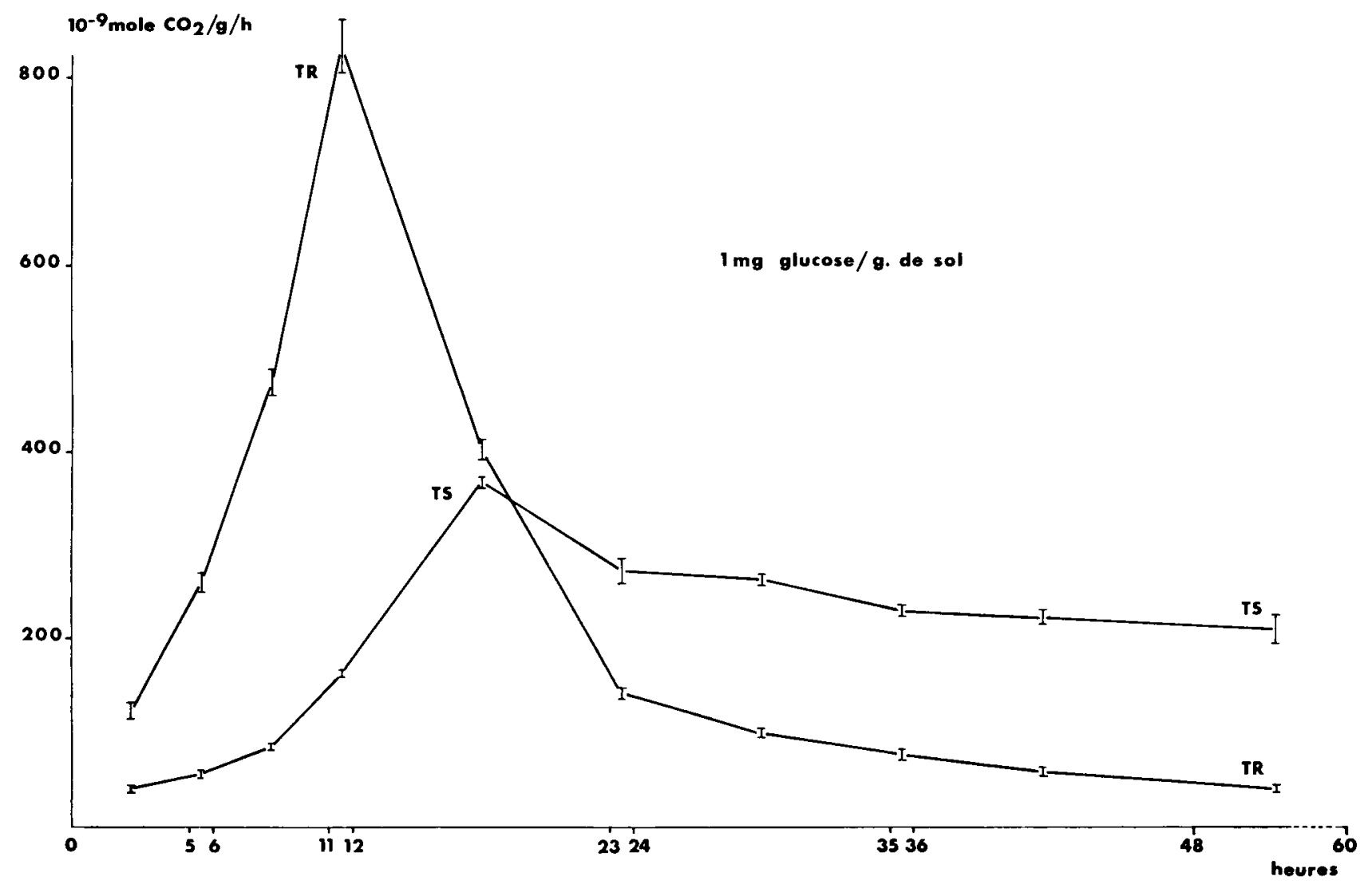

Figure 2

Cinétique du dégagement de $\mathrm{CO}_{2}$ par une terre résistante (TR) et par une terre sensible (TS) après addition de glucose (I $\mathrm{mg} / \mathrm{g}$ de terre).
Kinetics of $\mathrm{CO}_{2}$ release from suppressive (TR) and conducive (TS) soil after addition of $1 \mathrm{mg}$ of glucose per $\mathrm{g}$. 
Il en résulte que les microorganismes à phase de latence relativement longue et vitesse de croissance faible, en particulier les Fusarium, ont une possibilité moindre d'entrer en activité et de se développer à la suite d'un apport énergétique limité, constitué par les exsudats racinaires.

Dans le sol résistant, lorsque les conditions deviennent limitantes après quelques heures seulement d'intense activité microbienne, les chlamydospores de Fusarium n'ont pas eu le temps de donner naissance à un thalle important. Au contraire, dans le sol sensible, la croissance des tubes germinatifs se poursuit plus longtemps, ce qui augmente la probabilité de rencontre avec la surface racinaire.

Les résultats qui viennent d'être présentés et ceux qui concernent le comportement des populations de Fusarium après apport de glucose dans ces 2 sols (ALABOUVETTE et al., 1985) confortent l'hypothèse du rôle de la compétition nutritive non spécifique dans les mécanismes de résistance générale (general suppression) (COOK \& BAKER, 1983). Ils indiquent claire- ment que non seulement la biomasse mais aussi la capacité de la microflore à répondre rapidement à un apport nutritif sont des facteurs primordiaux qui conditionnent l'efficacité de ce type de résistance.

Mais la résistance des sols de Châteaurenard fait en outre appel à un mécanisme spécifique (specific suppression) basé sur l'activité d'une population particulière de microorganismes. Il a été démontré (ROUXEL et al., 1979) qu'elle met en jeu une compétition intragénérique entre formes pathogènes et formes saprophytes de Fusarium spp. qui permet d'expliquer sa spécificité d'action vis-à-vis des fusarioses vasculaires (AlABOUVETTE et al., 1980a). Ainsi, la résistance des sols de Châteaurenard serait la conséquence d'une compétition intragénérique entre Fusarium, étroitement conditionnée par l'intensité de la compétition générale qui est elle-même fonction de la masse et de l'activité de la microflore tellurique dans son ensemble.

Reçu le 12 avril 1984. Accepté le 22 août 1984.

\section{RÉFÉRENCES BIBLIOGRAPHIQUES}

\begin{abstract}
Alabouvette C., 1983. La réceptivité des sols aux fusarioses vasculaires. Rôle de la compétition nutritive entre microorganismes. Thèse Doct. ès-Sciences Naturelles, Univ. de Nancy, $158 \mathrm{p}$.
\end{abstract}

Alabouvette C., Couteaudier Y., Louvet J., 1985. Recherches sur la résistance des sols aux maladies. XI. - Etude comparative du comportement des Fusarium spp. dans un sol résistant et un sol sensible enrichis en glucose. Agronomie, 5 (1), 63-68.

Alabouvette C., Rouxel F., Louvet J., 1980a. Recherches sur la résistance des sols aux maladies. VI. Mise en évidence de la spécificité de la résistance d'un sol vis-à-vis des fusarioses vasculaires. Ann. Phytopathol., 12 (1), 11-19.

Alabouvette C., Rouxel F., Louvet J., 1980b. Recherches sur la résistance des sols aux maladies. VII. Etude comparative de la germination des chlamydospores de Fusarium oxysporum et Fusarium solani au contact de sol résistant et sensible aux fusarioses vasculaires. Ann. Phytopathol., 12 (1), 21-30.

Anderson J. P. E., Domsch K. H., 1978. A physiological method for the quantitative measurement of microbial biomass in soils. Soil Biol. Biochem., 10, 215-221.
Cook R. J., Baker K. F., 1983. The nature and practice of biological control of plant pathogens. Am. Phytopathol. Soc., St Paul MN, USA, $539 \mathrm{p}$.

Jenkinson D. S., Powelson D. S., 1976. The effects of biocidal treatments on metabolism in soil. V. A method for measuring soil biomass. Soil Biol. Biochem., 8, 209-213.

Lockwood J. L., 1981. Exploitation competition, 319-350. In D. T. Wicklow \& G. C. Carroll : "The Fungal Community. Its organization and role in the ecosystem ». M. Dekker, Inc., $833 \mathrm{p}$.

Mangenot F., Diem H. G., 1979. Fundamentals of biological control, 207-265. In S. Y. Krupa \& Y. R. Dommergues. « Ecology of root pathogens ». $280 \mathrm{p}$.

Rouxel F., Alabouvette C., Louvet J., 1979. Recherches sur la résistance des sols aux maladies. IV. Mise en évidence du rôle des Fusarium autochtones dans la résistance d'un sol à la fusariose vasculaire du melon. Ann. Phytopathol., 11, 199-207. 